\title{
Immersiveness and Physiological Arousal within Panoramic Video-Based Virtual Reality
}

\author{
MARY F. MACEDONIO, Psy.D., ${ }^{1}$ THOMAS D. PARSONS, Ph.D.,${ }^{2,3}$ \\ RAYMOND A. DIGIUSEPPE, Ph.D., ${ }^{1}$ BRENDA K. WEIDERHOLD, Ph.D., ${ }^{4}$ \\ and ALBERT A. RIZZO, Ph.D. ${ }^{2,3}$
}

\begin{abstract}
In this paper, we discuss findings from a study that used panoramic video-based virtual environments (PVVEs) to induce self-reported anger. The study assessed "immersiveness" and physiological correlates of anger arousal (i.e., heart rate, blood pressure, galvanic skin response [GSR], respiration, and skin temperature). Results indicate that over time, panoramic video-based virtual scenarios can be, at the very least, physiologically arousing. Further, it can be affirmed from the results that hypnotizability, as defined by the applied measures, interacts with group on physiological arousal measures. Hence, physiological arousal appeared to be moderated by participant hypnotizability and absorption levels.
\end{abstract}

\section{INTRODUCTION}

$\mathbf{R}$ ECENT ADVANCES in panoramic video camera systems have produced new methods for the creation of virtual environments. With these systems, users can capture, play back, and observe pictorially accurate 360-degree video scenes of "realworld" environments. When delivered via an immersive head-mounted display (HMD), an experience of presence within these captured scenarios can be supported in human users. Panoramic video camera systems may be used to create virtual environments that are immersive and invoke physiological response. In line with the theory of Foa and Kozak, ${ }^{1}$ participants need to feel "present" in the virtual environment, the virtual environment should elicit emotional response, and cognitive changes must be generalizable to real-world situations.

Exposure to emotional situations results in regular activation of cerebral metabolism in brain areas associated with inhibition of maladaptive associa- tive processes. ${ }^{2}$ Identical neural circuits have been found to be involved in affective regulation across affective disorders. ${ }^{3,4}$ Systematic and controlled therapeutic exposure to physiologically arousing stimuli may enhance emotional regulation through adjustments of inhibitory processes on the amygdala by the medial prefrontal cortex during exposure and through structural changes in the hippocampus after successful therapy. ${ }^{5}$

The value in using virtual reality (VR) technology to produce simulations targeting cognitive and behavioral clinical applications has been acknowledged by an encouraging body of research. $6,7,8,9,10,11$ Some of the work in this area has addressed anxiety disorders, ${ }^{12}$ pain distraction, ${ }^{13}$ posttraumatic stress disorder (PTSD), ${ }^{14}$ and such cognitive processes as attention, ${ }^{15}$ memory, ${ }^{16}$ executive functioning, ${ }^{17}$ and visuospatial abilities. ${ }^{18,19}$ VR offers the capacity for systematically delivering stimuli within an immersive virtual environment, thereby allowing for physiological response measurement to occur while a client is exposed to content that is more

\footnotetext{
${ }^{1}$ Department of Psychology, St. John's University, New York.

${ }^{2}$ Institute for Creative Technologies, University of Southern California, Marina del Rey, California.

${ }^{3}$ Institute for Creative Technologies and School of Gerontology, University of Southern California, Marina del Rey, California.

${ }^{4}$ Interactive Media Institute, San Diego, California.
} 
similar to the real world. Thus far, the recording of psychophysiological variables while participants operate within virtual environments has produced useful results in studies examining attention and presence. ${ }^{20,21}$ As such, the VR assets that allow for precise stimulus delivery within ecologically enhanced scenarios appears well matched for this research.

Researchers have found that the individual characteristics of study participants may impact the immersiveness and subsequent findings of a given study. Of primary import is the extent to which a participant is capable of "absorption" and "hypnotizability." 22 Hence, individual differences may moderate presence and confound findings. The propensity of participants to get involved passively in some activity and their ability to concentrate and block out distraction are important factors to consider when conducting a study. ${ }^{23}$ Likewise, evidence suggests that hypnotizability plays a role in the outcome of studies using VR. ${ }^{22}$ Research into these moderating individual traits is of value because such research may augment participant selection.

In this paper, we discuss findings from a study that used panoramic video-based virtual environments (PVVEs) to induce self-reported anger. The study assessed "immersiveness" and physiological correlates of anger arousal (i.e., heart rate, blood pressure, galvanic skin response [GSR], respiration, and skin temperature). Given the general consensus that a particular event or specific external agent, namely an individual, must be viewed as generating a negative event in order for anger to be evoked, ${ }^{24,25}$ this work focused on the use of PVVE scenarios that were captured in an office work environment that featured actors assuming the roles of hostile coworkers and administrators for use in anger arousal and management research. The presented findings report the impact of immersiveness on physiological arousal within a PVVE.

\section{METHOD}

\section{Participants}

Participants ultimately either were recruited by direct request or opted to participate after learning of the study from others engaged in the research process. The resulting sample included 41 participants (mean age $=40.3 ; S D=14.68 ; 56 \%$ female). Ethnicity distribution was as follows: Caucasian = $80 \%$; African American $=7 \%$; Hispanic $=10 \%$; and
Asian $=3 \%$. Participant work experiences included corporate, academic, medical, and independent environments.

\section{Instruments}

Psychological Measures. Participants completed a battery of self-report measures: the State-Trait Anger Expression Inventory 2 (STAXI-2) scale was used to assess anger; the Positive Affect Negative Affect Scale (PANAS) was used to assess affect; the Immersive Tendencies Questionnaire (ITQ) was used to assess presence; the Tellegen Absorption Scale and Openness to Experience Scale questionnaires were used to assess personality; and a visual analog scale (VAS) was used as an outcome measure of psychological change to identify perception of arousal (high, medium, and low).

Physiological Measures. Seven physiological measures were analyzed (systolic [SBP] and diastolic [DBP] blood pressure, mean arterial pressure $[\mathrm{MAP}]$, heart rate $[H R]$, respiration rate [RR], galvanic skin response [GSR], and temperature [T]) at the three distinct time periods of acclimation (point 2), midpoint (end of Scene 5), and endpoint (end of Scene 10), as reflected in the protocol process shown in Figure 1.

All physiological signals were recorded by a ProComp + unit distributed by Thought Technology Ltd. Heart rate was recorded using blood volume pressure (BVP) sensors placed on the fingertips of the index and ring fingers of the left hand using Velcro strips. Respiration rate was recorded using a strain gauge sensor positioned $3 \mathrm{~cm}$ above the umbilicus. GSR and temperature were recorded via sensors taped to the third and fifth fingers of the left hand, respectively. Panoramic video scenarios were delivered via the VFX 3D HMD (Interactive Imaging Systems, Ltd).

\section{Virtual Reality Scenario Example}

Each scene opens to reveal the participant's desk and computer (positioned on the left side of the panel); within a few seconds, his or her "virtual boss" arrives and addresses the participant in a fairly hostile and condescending fashion. In this graphically represented sample scenario, the virtual supervisor makes the following statement in an ever increasing and threatening tone:

Okay, so listen. We've been at this for six months now. We have customers waiting, we got products to ship, you're just not getting it done. We're get- 


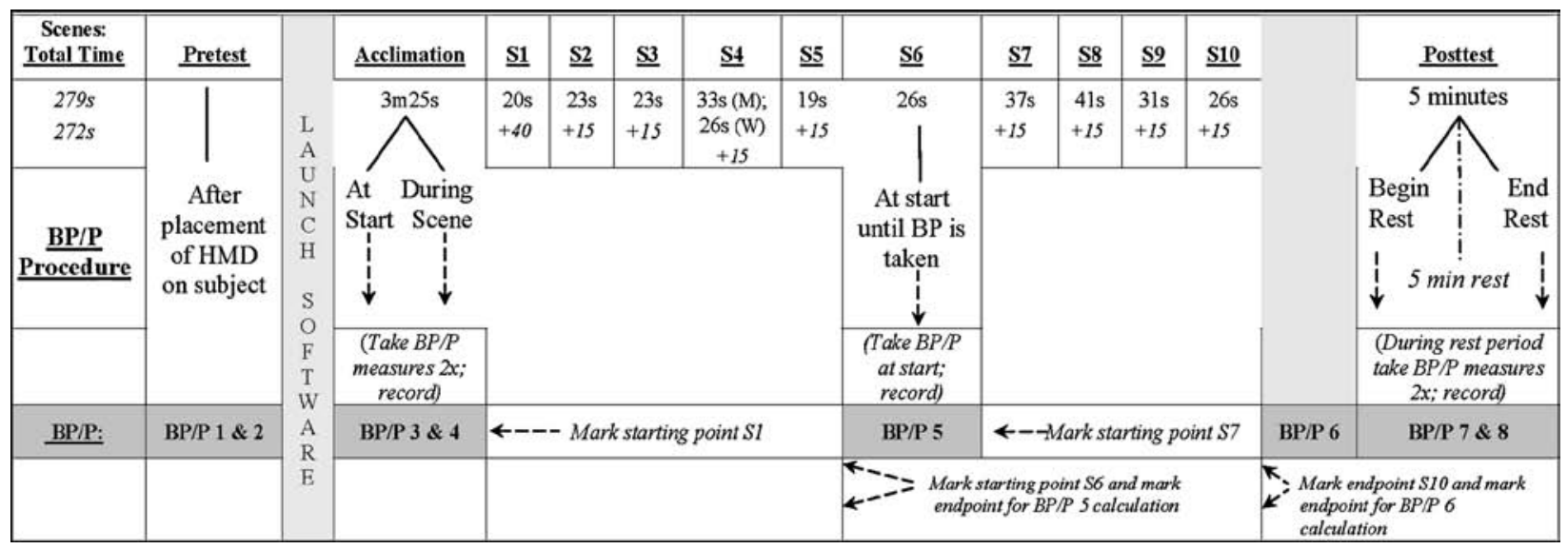

FIG. 1. Protocol process.

ting calls every other day from people. Now, what is it going to take? What do we have to do? If you can't make it, just let us know, and we're going to have to let you go ... you're gone! Okay? So, this Friday, it's either ship this product or you might as well ship your own stuff out the door, 'cause that's where you're going. Okay? Ya get it?

\section{Procedure}

All analyses were completed by an experienced examiner who worked directly with each participant. First, consent forms were collected and intakes performed for all participants. Next, participants completed a battery of self-report measures: STAXI-2 scale, PANAS, Immersive Tendencies Questionnaire, Tellegen Absorption Scale, openness to experience scale, and the VAS. Subsequently, participants were exposed to the virtual environment using a compilation of virtual scenes (concert, elevator ride, automobile drive, and university football game) to allow them to habituate to the virtual environment.

Prior to obtaining physiological measures, all participants were asked to rate their state anger from 1 (not angry) through 10 (extremely angry) to assist an understanding of how to rate their anger after being exposed to upcoming scenarios. No individuals were excluded regardless of their initial response. This process also assisted participants in understanding how to respond to the scenes.

A synopsis of the scenes was presented prior to testing, with each participant's blood pressure recorded twice using an Omron digital blood pressure monitor placed around the right arm. Temperature was measured by a probe placed on the fifth digit of the left hand, heart rate by reusable silverized Velcro electrodes placed on the index and ring fingers of the left hand, and skin conductance (GSR) by a Velcro light sensor probe placed on the middle finder of the left hand. Upon completion of procedural explanations, recording of initial blood pressure measures, and checks of all physiological recordings, the protocol process commenced. Placement of physiological measurement feeds was tested at the start and periodically inspected throughout testing to ensure neither shifting nor slippage of the electrodes. Participants were able to move their hands throughout the testing process because the length of the feeds comfortably allowed for ease of movement.

\section{RESULTS}

To analyze whether group (imagery group versus virtual reality group) measures changed significantly from each other at each time point, a series of three (time) by two (group) mixed analyses of variance (ANOVAs) were run on each of the five dependent variables. For some of the dependent measures (SBP, DBP, MAP, and HR) there were significant changes, with differences found between groups for all blood pressure measures across time, as noted in Table 1. However, no significant group by time interaction was found on any measure.

Table 2 presents results of changes across time in physiological measures by group. In the imagery group, significant results were obtained in SBP and MAP, with marginal significance found in DBP and HR, while the VR group showed significant changes across time in HR only, with moderate significant effects noted in SBP. At each time point, imagery group statistics showed significant SBP and MAP effects at both midpoint and endpoint, marginal sig- 
Table 1. Summary of Time $\times$ Group: Mixed ANOVAs

\begin{tabular}{|c|c|c|c|c|c|c|c|c|}
\hline & \multicolumn{3}{|c|}{ Change across time } & \multicolumn{2}{|c|}{ Difference between groups } & \multicolumn{3}{|c|}{ Group by time interaction } \\
\hline & $\begin{array}{l}\text { Wilks's } \\
\text { lambda }\end{array}$ & $\mathrm{F}$ & $\mathrm{p}$ & $\mathrm{F}$ & $\mathrm{p}$ & $\begin{array}{l}\text { Wilks's } \\
\text { lambda }\end{array}$ & $\mathrm{F}$ & $\mathrm{p}$ \\
\hline SBP & 0.698 & 16.442 & $<0.000$ & 6.659 & 0.012 & 0.959 & 1.606 & 0.207 \\
\hline$D B P$ & 0.919 & 3.331 & 0.041 & 7.853 & 0.006 & 0.976 & 0.932 & 0.398 \\
\hline MAP & 0.788 & 10.236 & $<0.001$ & 8.442 & 0.005 & 0.952 & 1.934 & 0.152 \\
\hline$H R$ & 0.739 & 12.357 & $<0.001$ & 0.023 & 0.879 & 0.942 & 2.150 & 0.124 \\
\hline$R R$ & 0.991 & 0.326 & 0.723 & 0.235 & 0.629 & 0.998 & 0.053 & 0.949 \\
\hline GSR & 0.990 & 0.359 & 0.700 & 0.308 & 0.581 & 0.993 & 0.258 & 0.773 \\
\hline$T$ & 0.993 & 0.217 & 0.805 & 0.003 & 0.958 & 0.954 & 1.513 & 0.228 \\
\hline
\end{tabular}

Seven physiological measures were analyzed: systolic (SBP) and diastolic (DBP) blood pressure; mean arterial pressure (MAP); heart rate (HR); respiration rate (RR); galvanic skin response (GSR); and temperature $(\mathrm{T})$.

nificance in HR at endpoint, and marginal changes in DBP and HR at each time point. The VR group showed significant results at both midpoint and endpoint in HR, with marginal results in SBP found at endpoint.

Subsequent regression analyses on the moderator variables associated with hypnotizability (Tellegen Absorption Scale, Immersion Tendency Questionnaire, and openness to experience scale) and anger (state anger, trait anger, and negative affect) were performed separately, with repeated measures collapsed into a change measure to assess interaction effects on physiological measures. Table 3 presents results of the Tellegen Absorption Scale on the relationship between group and physiological measures, suggesting that there is a slight moderating effect on the relation between group and SBP change at midpoint, with both groups showing small decreases in this measure with increased absorption. DBP change at endpoint was marginal for those in the imagery group but dropped significantly at endpoint as VR group participants became more absorbed. On the MAP measure, interaction is clear between group and hypnotizability in predicting change: moderate significant effects were achieved at both time points, with VR group participants displaying greater decreases over time than those in the imagery group. Changes in GSR were found at midpoint and endpoint, showing a similar pattern of effect within each group, namely, as absorption increased, those in the imagery group experienced increases of GSR change at both time points, whereas the VR group showed less change at each point. The consistency of effects in this measure suggests that, overall, individuals higher in absorption showed greater changes in arousal when exposed to imagery versus VR.

Table 2. Changes across Time in Physiological Measures by Group

\begin{tabular}{|c|c|c|c|c|c|c|c|c|c|c|c|c|}
\hline & \multicolumn{6}{|c|}{ Group 1 (Imagery) } & \multicolumn{6}{|c|}{ Group $2(V R)$} \\
\hline & \multirow{2}{*}{\multicolumn{3}{|c|}{$\begin{array}{c}\text { Mean } \\
\text { across time pts. }\end{array}$}} & \multicolumn{3}{|c|}{ Wilks's lambda } & \multirow{2}{*}{\multicolumn{3}{|c|}{$\begin{array}{l}\text { Mean } \\
\text { across time pts. }\end{array}$}} & \multicolumn{3}{|c|}{ Wilks's lambda } \\
\hline & & & & Value & F & $\mathrm{p}$ & & & & Value & $\mathrm{F}$ & $\mathrm{p}$ \\
\hline$R P$ & 130.4 & 137.2 & 138.8 & 0.450 & 22.00 & $<0.001$ & 121.1 & 124.2 & 126.4 & 0.853 & 3.36 & 0.045 \\
\hline$\supset B P$ & 80.1 & & & 0.781 & 5.04 & 0.0 & & & & 0.980 & 0.39 & 0.680 \\
\hline МAP & 96.9 & 101.1 & 102.8 & 0.56 & 13.88 & $<0.0$ & 90.2 & 91 & & 0.928 & 1.52 & 0.232 \\
\hline$H R$ & 68.0 & 74.4 & 75.3 & 0.7861 & 5.0 & & 69 & 76 & 77 & 0.695 & 8.12 & $<0.001$ \\
\hline$R R$ & 25.9 & 28.1 & 30.9 & 0.987 & 0.2 & & 21 & 19 & 25 & 0.993 & 0.1 & 0.883 \\
\hline GSI & 15.6 & 16.7 & 16.0 & & 0.5 & & & 15 & 15 & 0.999 & & 0.976 \\
\hline$T$ & 76.6 & 76.7 & 76.6 & 0.937 & 1.05 & 0.362 & 76.5 & 76.6 & 76.5 & 0.962 & 0.605 & 0.553 \\
\hline
\end{tabular}

Seven physiological measures were analyed: systolic (SBP) and diastolic (DBP) blood pressure; mean arterial pressure (MAP), heart rate (HR); respiration rate (RR); galvanic skin response (GSR); and temperture $(\mathrm{T})$. 
Table 3. Interaction EfFect of Tellegen Absorption Scale on Physiological Measures at Midpoint and Endpoint

\begin{tabular}{|c|c|c|c|c|c|c|c|c|c|c|}
\hline & \multicolumn{5}{|c|}{ Midpoint (S5) } & \multicolumn{5}{|c|}{ Endpoint (S10) } \\
\hline & $\begin{array}{l}R \text {-square } \\
\text { change }\end{array}$ & $\begin{array}{c}F \\
\text { change }\end{array}$ & $d f 1$ & $d f 2$ & $\begin{array}{l}\text { Sig. F } \\
\text { change }\end{array}$ & $\begin{array}{l}R \text {-square } \\
\text { change }\end{array}$ & $\begin{array}{c}F \\
\text { change }\end{array}$ & $d f 1$ & $d f 2$ & $\begin{array}{l}\text { Sig. F } \\
\text { change }\end{array}$ \\
\hline SBP & 0.039 & 3.22 & 1 & 75 & 0.077 & 0.032 & 2.63 & 1 & 75 & 0.109 \\
\hline$D B P$ & 0.032 & 2.55 & 1 & 75 & 0.115 & 0.044 & 3.69 & 1 & 75 & 0.058 \\
\hline MAP & 0.058 & 4.90 & 1 & 75 & 0.030 & 0.061 & 5.241 & 1 & 75 & 0.025 \\
\hline$H R$ & 0.002 & 0.114 & 1 & 69 & 0.737 & 0.000 & 0.026 & 1 & 69 & 0.872 \\
\hline$R R$ & 0.000 & 0.002 & 1 & 69 & 0.962 & 0.013 & 0.914 & 1 & 69 & 0.342 \\
\hline GSR & 0.042 & 3.079 & 1 & 69 & 0.084 & 0.078 & 6.098 & 1 & 69 & 0.016 \\
\hline$T$ & 0.015 & 0.984 & 1 & 62 & 0.325 & 0.001 & 0.065 & 1 & 62 & 0.800 \\
\hline
\end{tabular}

Seven physiological measures were analyzed: systolic (SBP) and diastolic (DBP) blood pressure; mean arterial pressure (MAP); heart rate (HR); respiration rate (RR); galvanic skin response (GSR); and temperature $(\mathrm{T})$.

Results of whether the openness to experience domain moderates the relationship between group and physiological measures suggest a moderating effect on the relation between group and both DBP change and MAP change at both midpoint and endpoint. There also exists a slight moderating effect on the relation between group and temperature change at midpoint. Results are presented in Table 4.

Descriptive statistics of subjects' VAS results of subjective anger experienced across the sample are reported in Table 5 and reveal a trend of consistent increases across scenario delivery overall. This result suggests that participants' perception of the scenes, whether using imagery or a virtual environment, induced anger responses.

A series of 13 (time) by 2 (group) mixed ANOVAs were run at each time point to further evaluate whether group measures changed significantly from each other; results are presented in Table 6. Restricted attention was paid to the lower order trends based on the visual inspection of the results, which revealed a significant linear (low to high), quadratic (angry faster, leveled off), and cubic trend (angry fast, leveled off, and declined again at posttest). Therefore, both imagery and VR seemed to increase participants' subjective experiences of anger. However, VAS results reveal an overall effect of time that was not significantly different for the two groups.

\section{DISCUSSION}

Results indicate that both groups showed changes over time, although not across all physio-

Table 4. Interaction Effect of Openness to Experience on Physiological Measures at Midpoint and Endpoint

\begin{tabular}{|c|c|c|c|c|c|c|c|c|c|c|}
\hline & \multicolumn{5}{|c|}{ Midpoint (S1-S5) } & \multicolumn{5}{|c|}{ Endpoint (S6-S10) } \\
\hline & $\begin{array}{l}R \text {-square } \\
\text { change }\end{array}$ & $\begin{array}{c}F \\
\text { change }\end{array}$ & $d f 1$ & $d f 2$ & $\begin{array}{l}\text { Sig. F } \\
\text { change }\end{array}$ & $\begin{array}{l}\text { R-square } \\
\text { change }\end{array}$ & $\begin{array}{c}F \\
\text { change }\end{array}$ & $d f 1$ & $d f 2$ & $\begin{array}{l}\text { Sig. F } \\
\text { change }\end{array}$ \\
\hline$S B P$ & 0.028 & 2.23 & 1 & 75 & 0.140 & 0.005 & 0.39 & 1 & 75 & 0.532 \\
\hline$D B P$ & 0.057 & 4.65 & 1 & 75 & 0.034 & 0.047 & 3.80 & 1 & 75 & 0.055 \\
\hline MAP & 0.076 & 6.45 & 1 & 75 & 0.013 & 0.044 & 3.55 & 1 & 75 & 0.063 \\
\hline$H R$ & 0.004 & 0.294 & 1 & 69 & 0.590 & 0.005 & 0.345 & 1 & 69 & 0.559 \\
\hline$R R$ & 0.006 & 0.450 & 1 & 69 & 0.505 & 0.004 & 0.262 & 1 & 69 & 0.611 \\
\hline GSR & 0.027 & 1.960 & 1 & 69 & 0.166 & 0.001 & 0.083 & 1 & 69 & 0.774 \\
\hline$T$ & 0.072 & 5.118 & 1 & 62 & 0.027 & 0.006 & 0.393 & 1 & 62 & 0.533 \\
\hline
\end{tabular}

Seven physiological measures were analyzed: systolic (SBP) and diastolic (DBP) blood pressure; mean arterial pressure (MAP); heart rate (HR); respiration rate (RR); galvanic skin response (GSR); and temperature $(\mathrm{T})$. 
Table 5. VAS Descriptive Statistics at Each Time Point on Sample

\begin{tabular}{lccccccccc}
\hline & \multicolumn{4}{c}{ Sample } & & & \multicolumn{3}{c}{ Sample } \\
& Mean & SD & Min & Max & & Mean & SD & Min & Max \\
\hline VAS at pretest & 1.09 & 0.288 & 1 & 2 & VAS at scene 5 & 6.09 & 2.864 & 1 & 10 \\
VAS at acclimation & .04 & 0.208 & 1 & 2 & VAS at scene 6 & 5.69 & 2.664 & 1 & 10 \\
VAS at scene 1 & 4.03 & 2.424 & 1 & 10 & VAS at scene 7 & 5.87 & 2.785 & 1 & 10 \\
VAS at scene 2 & 4.42 & 2.381 & 1 & 10 & VAS at scene 8 & 6.09 & 2.778 & 1 & 10 \\
VAS at scene 3 & 5.25 & 2.665 & 1 & 10 & VAS at scene 9 & 6.13 & 2.510 & 1 & 10 \\
VAS at scene 4 & 4.90 & 3.149 & 1 & 10 & VAS at scene 10 & 7.49 & 2.648 & 1 & 10 \\
& & & & & VAS at posttest & 2.76 & 2.310 & 1 & 10 \\
\hline
\end{tabular}

VAS, Visual Analog Scale.

logical measures. Results tended to follow the anticipated pattern of measures rising at each time point both across the sample and between groups, with GSR and temperature remaining relatively stable. This finding suggests that PVVE anger scenarios can be, at the very least, physiologically arousing. Further, it can be affirmed from the results that hypnotizability, as defined by the applied measures, interacts with group on physiological arousal measures. Hence, physiological arousal appeared to be moderated by participant hypnotizability and absorption levels.

Within the first class of individual difference variables, PVVEs appeared to have a nonarousing effect on those who were highly absorbed. High-absorption individuals may be capable of imagining and becoming angry when it is suggested, thereby not requiring PVVE immersion to assist them. Indeed, the results suggest that PVVEs may actually be distracting to individuals higher in absorption. MAP was affected at both time points in similar ways for both groups on the openness to experience scale, showing initially slight increases at midpoint with moderate decreases at endpoint. As participants increased in openness to the scenes, it is possible that they acclimated to the content that affected their physiological arousal. It can be affirmed from the results that hypnotizability, as defined by the applied measures, interacts with group on the arousal measure of blood pressure in only two of three scales, with some restricted effects seen in GSR and temperature.

Measures of anger and negative affect and blood pressure change across midpoint and endpoint were found in both state and trait anger, with heart rate being affected at both time points in negative affect. Although MAP increased and then leveled off for those in the imagery group, it consistently

Table 6. VAS Results at Each Time Point by Group

\begin{tabular}{lccccccrr}
\hline & \multicolumn{3}{c}{ Group 1 (Imagery) } & \multicolumn{5}{c}{ Group 2(VR) } \\
& Mean & SD & Min & Max & Mean & SD & Min & Max \\
\hline VAS at pretest & 1.03 & 0.177 & 1 & 2 & 1.14 & 0.355 & 1 & 2 \\
VAS at acclimation & 1.03 & 0.177 & 1 & 2 & 1.06 & 0.236 & 1 & 2 \\
VAS at scene 1 & 4.06 & 2.015 & 1 & 8 & 4.00 & 2.776 & 1 & 10 \\
VAS at scene 2 & 4.25 & 2.436 & 1 & 10 & 4.57 & 2.355 & 1 & 9 \\
VAS at scene 3 & 5.50 & 2.940 & 1 & 10 & 5.03 & 2.407 & 1 & 10 \\
VAS at scene 4 & 4.47 & 3.253 & 1 & 9 & 5.29 & 3.044 & 1 & 10 \\
VAS at scene 5 & 6.00 & 3.121 & 1 & 10 & 6.17 & 2.651 & 1 & 10 \\
VAS at scene 6 & 5.22 & 2.802 & 1 & 10 & 6.11 & 2.494 & 1 & 10 \\
VAS at scene 7 & 6.09 & 2.900 & 1 & 10 & 5.66 & 2.700 & 1 & 10 \\
VAS at scene 8 & 6.22 & 3.035 & 1 & 10 & 5.97 & 2.561 & 1 & 10 \\
VAS at scene 9 & 6.09 & 2.692 & 1 & 10 & 6.17 & 2.370 & 1 & 10 \\
VAS at scene 10 & 7.13 & 2.927 & 1 & 10 & 7.83 & 2.358 & 2 & 10 \\
VAS at posttest & 2.47 & 1.900 & 1 & 10 & 3.03 & 2.629 & 1 & 10 \\
\hline
\end{tabular}

VAS, Visual Analog Scale. 
decreased across time on state-anger scores for VR group participants. While this unanticipated result denotes that the amount of increase in blood pressure change was smaller for those in the VR group than in the imagery group, close analysis reveals a rather skewed anger distribution: almost all participants scored very low on this scale as well. Additionally, there were consistent decreases across time in this physiological measure as it applies to trait anger, with only systolic blood pressure increasing at midpoint for those in panoramic the PVVE. From these results, it is likely that the moderating effect of anger cannot be effectively examined, because an angry person who is, for example, high in immersion may respond differently to VR than a person who scored low on the scale. In contrast, a person with high trait anger and absorption may benefit from VR, whereas another with low absorption scores may respond to imaginal techniques. Considering that anger is further moderated by a person's level of hypnotizability and absorption, some evidence, albeit weak, exists that absorption may be an important variable for choosing between VR and imagery techniques in a therapeutic setting.

To the credit of the scenarios developed for this study, at least 3 of the 10 most anger-provoking factors as defined by Mabel ${ }^{26}$ were considered to have been effectively presented: experiencing personal degradation or unfair treatment (and being powerless to stop it); being treated unfairly, unkindly, or in a prejudicial way whether or not one is present; and being the object of verbal or physical assault. However, considering that many anger reactions are generated not by what is said but by how it is communicated (such as voice intonation, invasion of personal space, facial expression), one may argue that only one of the 10 scenes presented offered each of these elements. In retrospect, many subjects commented on a particular scene, complaining that the individual presenting the negative commentary was too much "in my face," which seemed to engender a greater anger response than did the others. Perhaps future research should include facial closeups that clearly display contemptuous connotations and undermining statements to effectively provoke an angry response, as opposed to full-body scenarios. Additionally, it is advised that subjects be offered a longer acclimation period to the environment in which the anger-engendering stimuli is presented so as to limit the amount of distraction that may occur when initially immersed into the settings.

An important limitation of the current study is that participants were neither clinically angry nor easily able to relate to the scenarios presented. Most of the subjects were employed outside the corporate sector, on which the scenarios were based, and when questioned upon completion of the study, admitted that very few scenes were individually relevant. Further, the number of subjects participating in this study may have been not only inappropriate given the lack of a clinically angry sample but also too small to truly test any meaningful factors concerning anger. A larger sample to test the hypotheses would have been optimal.

In summary, this preliminary study using a PVVE found absorption and hypnotizability to impact measures of physiological arousal. Future studies should make use of physiological data correlated with measures of immersion to augment and quantify the effects of PVVE scenarios.

\section{REFERENCES}

1. Foa, E.B., \& Kozak, M.J. (1986). Emotional processing of fear: exposure to corrective information. Psychological Bulletin 99:20-35.

2. Schwartz, J.M. (1998). Neuroanatomical aspects of cognitive-behavioural therapy response in obsessivecompulsive disorder. An evolving perspective on brain and behaviour. British Journal of Psychiatry Supplemental pp. 38-44.

3. De Raedt, R. (2006). Does neuroscience hold promise for the further development of behavior therapy? The case of emotional change after exposure in anxiety and depression. Scandinavian Journal of Psychology 47:225-236.

4. Mineka, S., Watson, D., \& Clark, L.A. (1998). Comorbidity of anxiety and unipolar mood disorders. Annual Review of Psychology 49:377-412.

5. Hariri, A.R., Bookheimer, S.Y., \& Mazziotta, J.C. (2000). Modulating emotional responses: effects of a neocortical network on the limbic system. Neuroreport 11:43-48.

6. Parsons, T.D., Rizzo, A.A., van der Zaag, C., McGee, J.S., \& Buckwalter, J.G. (2005). Gender and cognitive performance: a test of the common cause hypothesis. Aging, Neuropsychology, and Cognition 12:78-88.

7. Rizzo, A., \& Buckwalter, J.G. (1997). Virtual reality and cognitive assessment and rehabilitation: the state of the art. In Riva, G. (ed.) Virtual reality in neuro-psycho-physiology: cognitive, clinical, and methodological issues in assessment and rehabilitation. Amsterdam: IOS Press, pp. 123-146.

8. Rizzo, A.A., Buckwalter, J.G., Neumann, U., Kesselman, C., \& Thiebaux, M. (1998). Basic issues in the application of virtual reality for the assessment and rehabilitation of cognitive impairments and functional disabilities. CyberPsychology \& Behavior 1:59-78.

9. Rizzo, A. (2000). The virtual classroom: a virtual reality environment for the assessment and rehabilitation of attention deficits. CyberPsychology $\mathcal{E}$ Behavior 3:483-499. 
10. Rizzo, A.A., Buckwalter, J.G., \& van der Zaag, C. (2002). Virtual environment applications in clinical neuropsychology. In Stanney, K. (ed.) The handbook of virtual environments. Erlbaum, New York, pp. 1027-1064.

11. Rose, F.D., Brooks, B.M., \& Rizzo, A.A. (2005).Virtual reality in brain damage rehabilitation: review (Discussion, 263-271). CyberPsychology E Behavior 8:241262.

12. Krijn, M., Emmelkamp, P.M., Olafsson, R.P., \& Biemond, R. (2004). Virtual reality exposure therapy of anxiety disorders: a review. Clinical Psychology Review 24:259-281.

13. Gold, J.I., Kim, S.H., Kant, A.J., Joseph, M.H., \& Rizzo, A.A. (2006). Effectiveness of virtual reality for pediatric pain distraction during IV placement. CyberPsychology \& Behavior 9:207-213.

14. Rizzo, A.A., Pair, J., McNerney, P.J., Eastlund, E., Manson, B., Gratch, J., Hill, R., Roy, M., \& Swartout, B. (2005). Design and development of a VR therapy application for Iraq war veterans with PTSD. In Westwood, J.D., et al. (eds.) Technology and informatics, 111. Amsterdam: IOS Press. pp. 407-413.

15. Parsons, T.D., Bowerly, T., Buckwalter, J.G., \& Rizzo, A.A. (in press). A controlled clinical comparison of attention performance in children with ADHD in a virtual reality classroom compared to standard neuropsychological methods. Child Neuropsychology.

16. Matheis, R., Schultheis, M.T., Tiersky, L.A. , DeLuca, J., Mills, S.R., \& Rizzo, A.A. (in press). Is learning and memory different in a virtual environment? Journal of Clinical and Experimental Neuropsychology.

17. Rizzo, A.A., Klimchuk, D., Mitura, R., Bowerly, T., Buckwalter, J.G., \& Parsons, T. (2006). A virtual reality scenario for all seasons: the virtual classroom. CNS Spectrums 11:35-44.

18. Parsons, T.D., Larson, P. Kratz, K., Thiebaux, M., Bluestein, B., Buckwalter, J.G., \& Rizzo, A.A. (2004). Sex differences in mental rotation and spatial rotation in a virtual environment. Neuropsychologia 42:555-562.
19. Parsons, T.D., Rizzo, A.A., \& Buckwalter, J.G. (2004). Backpropagation and regression: comparative utility for neuropsychologists. Journal of Clinical and Experimental Neuropsychology 26:95-104.

20. Meehan, M., Insko, B., Whitton, M., \& Brooks, F. (2002). Physiological measures of presence in virtual environments. In proceedings of 4th Annual Presence Workshop, Philadelphia, May 2002.

21. Pugnetti, L., Meehan, M., \& Mendozzi, L. (2001). Psychophysiological correlates of virtual reality: a review. Presence 10:384-400.

22. Wiederhold, B.K., \& Wiederhold, M.D. (2000). Lessons learned from 600 virtual reality sessions. CyberPsychology \& Behavior 3:393-400.

23. Witmer, B.G., \& Singer, M.J. (1998). Measuring Presence in virtual environments: a presence questionnaire. Presence Teleoperators and Virtual Environments 7:225-240.

24. Smith C.A., \& Ellsworth, P.C. (1985). Patterns of cognitive appraisal in emotion. Journal of Personality and Social Psychology 48:813-838.

25. DiGiuseppe, R. (1999). End piece: reflections on the treatment of anger. Clinical Psychology 55, 365-379.

26. Mabel, S. (1994). Empirical determination of anger provoking characteristics intrinsic to provoking circumstances. Journal of Social \& Clinical Anger Psychology 13:174-188.

Address reprint requests to: Dr. Thomas D. Parsons Research Scientist, Neuropsychologist Institute for Creative Technologies University of Southern California 13274 Fiji Way, Office 301 Marina del Rey, CA, 90292-4019

E-mail: tparsons@usc.edu 